\title{
Primary tumor resection improves the survival of younger patients with metastatic breast cancer
}

\author{
TADAHIKO SHIEN $^{1,2}$, TAKAYUKI KINOSHITA ${ }^{1}$, CHIKAKO SHIMIZU $^{3}$, TAKASHI HOJO ${ }^{1}$, \\ NARUTO TAIRA $^{2}$, HIROYOSHI DOIHARA ${ }^{2}$ and SADAKO AKASHI-TANAKA ${ }^{1}$
}

\author{
${ }^{1}$ Department of Breast Surgery, National Cancer Center Hospital; ${ }^{2}$ Department of Cancer and Thoracic Surgery, \\ Okayama University; ${ }^{3}$ Breast and Medical Oncology Division, National Cancer Center Hospital, Okayama, Japan
}

Received September 23,2009; Accepted December 29, 2008

DOI: 10.3892/or_00000291

\begin{abstract}
Current treatments for metastatic breast cancer (MBC) include palliation with chemotherapy and/or hormone therapy, neither of which has the effect of adequately improving survival. Local surgery to remove the primary breast tumor is performed to improve local control and prevent uncontrolled chest wall disease (UCD). From June 1962 to February 2007, 344 patients with MBC were treated at National Cancer Center Hospital. In our review of these cases, we evaluated the prognostic impact of local surgery and other clinicopathological features. One hundred and sixty patients $(47 \%)$ underwent resection of primary breast tumor, while 184 $(53 \%)$ patients were treated without surgery. Overall survival (OS) was prolonged in patients treated with surgery $(\mathrm{p}=0.049)$, younger patients (age $<50, \mathrm{p}=0.023$ ), and patients with bone or soft tissue metastases $(\mathrm{p}=0.013)$. While surgery significantly improved OS in young patients $(\mathrm{p}=0.021)$, it did not increase OS in older patients (age $>51, \mathrm{p}=0.665$ ) or patients with visceral metastasis $(\mathrm{p}=0.797)$. This study demonstrated that local surgery improved OS of patients with MBC; local surgery should therefore be considered, especially in young patients. Prospective studies are required to validate these findings and evaluate the impact of surgical intervention.
\end{abstract}

\section{Introduction}

Recently, breast cancer became the most common cancer in Japanese women; and its incidence continues to increase. The incidence of metastatic breast cancer (MBC), defined as a primary breast tumor with distant metastasis, is increasing, comprising $~ 3 \%$ of newly diagnosed breast cancers in Japan, which is similar to the $6 \%$ seen in the United States according to the Surveillance, Epidemiology, and End Results (SEER)

Correspondence to: Dr Tadahiko Shien, Department of Cancer and Thoracic Surgery, Okayama University, 1-5-2 Shikata-cho, Okayama-shi, Okayama 700-8558, Japan

E-mail: tshien@md.okayama-u.ac.jp

Key words: stage IV, breast, surgery data. Treatment for breast cancer has also been progressing rapidly. Surgical interventions have become significantly less invasive with the introduction of breast-conserving therapy and sentinel lymph node biopsy; systemic chemotherapeutic agents have become increasingly safe and effective. Although such treatments have made better control of MBC possible, the therapeutic guidelines for MBC have not changed. Palliative treatment remains standard care, utilizing systemic therapy with chemotherapeutic, hormonal, and biologic agents $(1,2)$. Resection of the primary tumor was not considered curative treatment; it has been used solely as local therapy to prevent uncontrolled wall disease. Therefore, local surgery was performed relatively late in treatment and only if the primary tumor and metastases could not be reduced and controlled with systemic therapy.

The possibility that surgical procedures improve the survival of those patients has been reported retrospectively (3-6); this issue is still hotly debated at major breast conferences. The details of these studies, such as tumor sensitivity to systemic therapy and timing of surgery with respect to systemic treatment, were unclear. Improvements in primary systemic therapies have increased the numbers of MBC patients with resectable small primary tumors and controllable metastatic lesions after treatment. With all of these new developments, we need definitive guidelines for the treatment of these patients. In this study, we evaluate the efficacy of primary tumor resection at prolonging the overall survival of MBC patients and analyzed the relationship between response to surgery and clinicopathological features.

\section{Patients and methods}

Patients and treatments. Records of all patients with metastatic breast cancer (MBC) treated between June 1962 and February 2007 at the National Cancer Center Hospital (NCCH) was extracted from the database for inclusion in this retrospective study. Baseline information collected included patient demographics, tumor characteristics (size, node status, histological characteristics, estrogen and progesterone receptor status, and Her2/neu status), tumor site, number of metastases, type and timing of operative intervention, and use of hormonal therapy and chemotherapy. We classified patients into two categories based on the age when primary treatment began; 
younger patient was defined as $<50$ years old, while older included patients $>51$ years old. Sites of metastases were categorized as bone and/or soft tissue (bone, lymph nodes) or visceral (lungs, pleura, mediastinum, peritoneum, liver, and brain) metastases. All patients were treated with some form of systemic therapy, including chemotherapy and/or hormonal therapy.

In later years of the study (only after 2002), trastuzumab was administered to patients with tumors exhibiting HER2 overexpression. In the group who underwent surgery, surgical procedures included Halstead operation, modified radical mastectomy and breast conserving surgery with axillary dissection and simple mastectomy without axillary dissection. All primary breast tumors were removed completely. Time to surgery was calculated from the date in which primary treatment begun.

Metastatic involvement was determined by physical examination, biochemical analysis, and initial routine imaging procedures before or within one month of beginning primary treatment. Bone scans alone were not considered diagnostic of bone involvement. Abnormalities seen on bone scan were confirmed by radiography. Liver involvement was determined by computed tomography or ultrasound findings consistent with metastases. Pleural or peritoneal involvement was determined by positive cytology of effusion fluid and appropriate imaging studies. Cervical or contralateral lymph node involvement was classified as distant soft tissue metastases. Chest wall recurrence was excluded from soft tissue metastases.

Evaluation of pathological factors. Surgical specimens were sectioned at 7-10 $\mathrm{mm}$ for evaluation of the pathological response by pathologists. Expression levels of ER (1D5, Dako Cytomation), PgR (1A6, Novocastra), and HER2 (HercepTest $^{\circledR}$, Dako Cytomation) were examined by immunohistological staining. ER and PgR were classed as positive when $>10 \%$ of cancer cell nuclei exhibited positive staining, regardless of intensity. HER2 was scored as follows: (0), negative for cells; $(1+)$, slightly positive in $>10 \%$ of cancer cells; $(2+)$, moderately positive in $>10 \%$ of cancer cells; and $(3+)$, markedly positive in $>10 \%$ of cancer cells. Immunohistochemistry (IHC) with scores of $(2+)$ or $(3+)$ were defined as HER2-positive.

Statistical analysis. Overall survival (OS) was calculated from the date upon which treatment was initiated to the date of death or last visit. Kaplan-Meier plots and log-rank test were used to assess differences in survival. All comparisons were two-tailed. Cox-proportional hazards models were fit for OS. $\mathrm{P}<0.05$ were considered statistically significant.

\section{Results}

The medical records of $344 \mathrm{MBC}$ patients treated at $\mathrm{NCCH}$ were reviewed in this study. Table I lists patient characteristics. The median age at initiation of primary treatment was 54 years (28-82). We evaluated 141 (41\%) young patients $<50$ years of age and $203(59 \%)$ older patients $>51$ years of age. Sixty-six (19\%) patients were diagnosed between 1962-1980, 62 (18\%) between 1981-1990, 96 (28\%) between 1991-2000,
Table I. Patient characteristics and Cox proportional hazard model for overall survival.

\begin{tabular}{|c|c|c|}
\hline Parameters & $\begin{array}{l}\text { No. of patients } \\
(\%)\end{array}$ & $\begin{array}{l}\text { Hazard ratio } \\
(95 \% \mathrm{CI})\end{array}$ \\
\hline Age, median (range) & $54(28-82)$ & \\
\hline$\geq 51$ & $203(59)$ & 1.00 \\
\hline$\leq 50$ & $141(41)$ & $0.87(0.77-0.98)$ \\
\hline \multicolumn{3}{|l|}{ Period of diagnosis } \\
\hline $1962-1980$ & $66(19)$ & 1.00 \\
\hline 1981-1990 & $62(18)$ & $0.87(0.69-1.07)$ \\
\hline $1991-2000$ & $96(28)$ & $0.95(0.78-1.15)$ \\
\hline 2001-2007 & $120(35)$ & $0.85(0.69-1.03)$ \\
\hline \multicolumn{3}{|l|}{ Clinical T stage } \\
\hline $\mathrm{T} 1$ & $23(6)$ & 1.00 \\
\hline $\mathrm{T} 2$ & $60(17)$ & $0.92(0.65-1.41)$ \\
\hline $\mathrm{T} 3$ & $53(15)$ & $1.01(0.70-1.54)$ \\
\hline $\mathrm{T} 4$ & $208(60)$ & $1.11(0.82-1.63)$ \\
\hline
\end{tabular}

Estrogen receptor

Positive $106(31) \quad 1.00$

Negative $100(29)$

$1.12(0.93-1.33)$

Unknown

$138(40)$

$1.25(1.07-1.46)$

Progesterone receptor

Positive

$87(25)$

1.00

Negative

$120(35)$

$1.10(0.92-1.30)$

Unknown

137 (40)

$1.28(1.10-1.50)$

HER2

Positive 84 (24)

$111(32)$

149 (43)

1.00

Negative

$0.96(0.80-1.14)$

Unknown

$1.13(0.96-1.31)$

Site of metastases

Bone/soft tissue

169 (49)

1.00

Visceral

175 (51)

$1.16(1.03-1.29)$

Chemotherapy

Yes $315(88)$

1.00

No

29 (12)

$1.21(0.96-1.48)$

Hormone therapy

$\begin{array}{lll}\text { Yes } & 172(50) & 1.00\end{array}$

No $146(42)$

$0.90(0.75-1.09)$

Unknown

$26(8)$

$1.64(1.22-2.13)$

Local surgery

No $184(53)$

1.00

Yes $160(47)$

$0.89(0.79-1.00)$

and $120(35 \%)$ between 2001-2007. Clinical tumor size at diagnosis was assessed as T1 in $21(6 \%), \mathrm{T} 2$ in 60 (17\%), T3 in $53(15 \%)$, and T4 in $208(60 \%)$ patients. ER, PgR, and HER2 positivity was detected in $106(31 \%), 87$ (25\%), and $84(24 \%)$ patients, respectively. The ER/PgR and HER2 status of $137(40 \%)$ and $149(43 \%)$ patients, respectively, 
were unknown. Bone and/or soft tissue and visceral metastases were present in $169(49 \%)$ and 174 (51\%) patients, respectively.

Three hundred and fifteen $(88 \%)$ patients received chemotherapy, while $172(50 \%)$ patients received hormonal therapy. Local surgery was performed for 160 (47\%) patients. Surgical procedures included Halstead operation $(n=101,63 \%)$, modified radical mastectomy $(n=34,21 \%)$ and breast conserving surgery $(n=4,3 \%)$ with axillary dissection, and 21 patients $(13 \%)$ underwent simple mastectomy without axillary dissection. All primary breast tumors were removed completely. One hundred and fifty (94\%) of which underwent local surgery as primary therapy. The other patients underwent local surgery to avoid uncontrolled chest disease at late period of treatment when the primary tumors were regrowing. Local radiation after surgery was not used. There were patients without local surgery who underwent local radiation therapy.

Median follow-up time was 33 months (95\% confidence interval, 29.2-38.0 months). We plotted overall survival on Kaplan-Meier curves of the patient cohort according to each parameter (Fig. 1). OS was significantly prolonged in patients receiving surgery [surgery vs. no surgery, median survival time (MST): 27 vs. 22 months, $\mathrm{p}=0.049$ ], younger patients (younger vs. older, MST: 28 vs. 22 months, $\mathrm{p}=0.023$ ), and patients with bone/soft tissue metastasis (bone/soft tissue vs. visceral, MST: 29 vs. 21 months, $\mathrm{p}=0.013$ ). Hormonal therapy was also associated with improved OS (Fig. 1). Patients receiving hormonal therapy had a better prognosis than those who did not receive hormonal therapy. Chemotherapy was not associated with an improved OS. ER, PgR, and HER2 status, clinical tumor size, and period of diagnosis had no significant effects on OS (Table I).

The demographics and tumor characteristics of MBC patients treated with or without surgery are compared in Table II. Patients who underwent surgery tended to be younger $(p=0.02)$ and were diagnosed earlier in the study period $(\mathrm{p}<0.0001)$ than patients who did not undergo surgery. Clinical tumor size did not differ between the two groups $(\mathrm{p}=0.39)$. Patients with bone/soft metastasis $(\mathrm{p}<0.0001)$ or those who received hormonal therapy $(\mathrm{p}=0.05)$ were more likely to undergo surgery. There was no significant factor to predict survival in multivariate analysis (data not shown).

Fig. 2 displays Kaplan-Meier curves describing the OS of patient cohorts who received local surgery or no surgery as classified according to age and site of metastases. Surgery was associated with a better prognosis in younger patients (surgery vs. no surgery, MST: 35 vs. 24 months, $\mathrm{p}=0.021$ ). However, local surgery did not improve OS in older patients $(\mathrm{p}=0.665)$ and those with visceral metastases $(\mathrm{p}=0.797)$ and bone/soft tissue metastasis $(\mathrm{p}=0.095)$.

\section{Discussion}

The treatment of MBC has traditionally been palliative care with chemotherapy, hormonal therapy, and radiation therapy. According to the Hortobagyi algorithm (7), hormonal therapy is chosen as the first therapy for hormone receptorpositive MBC without visceral metastases. If MBC is hormone receptor-negative or resistant to hormone therapy, chemotherapy is used, but has the possibility of severely

\section{A. Comparison between local surgery and no surgery}

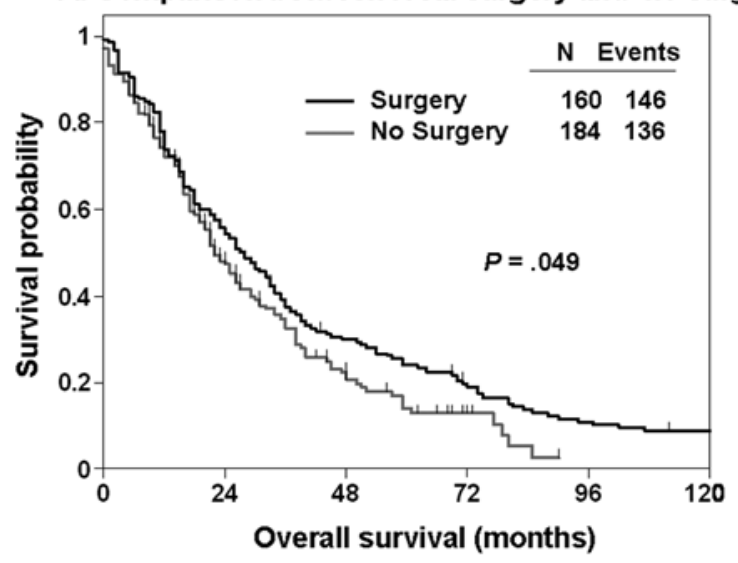

B. Comparison between younger $(<50)$ and older patients $(>51)$.

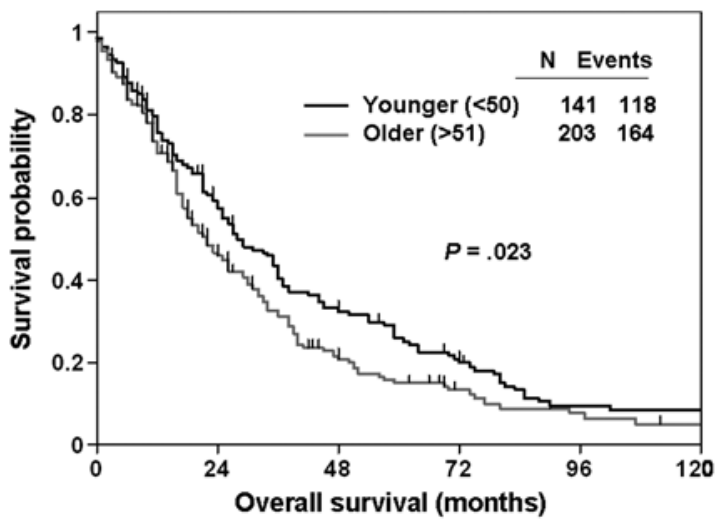

C. Comparison between Bone/soft tissue and visceral metastases.

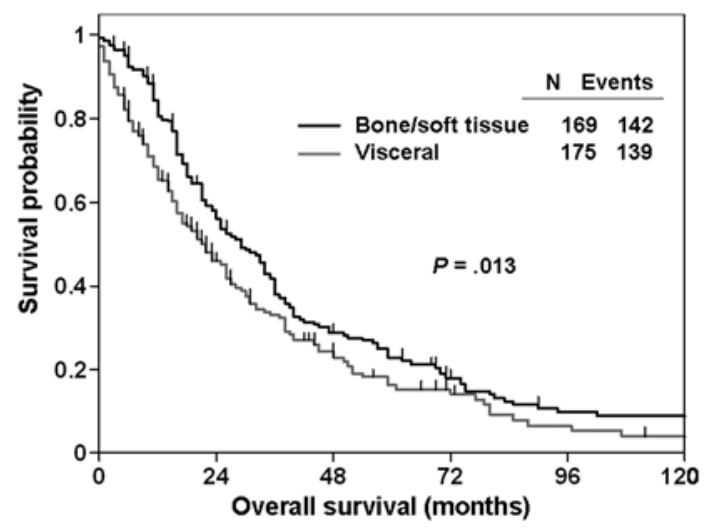

D. Comparison between hormone therapy and no hormone therapy.

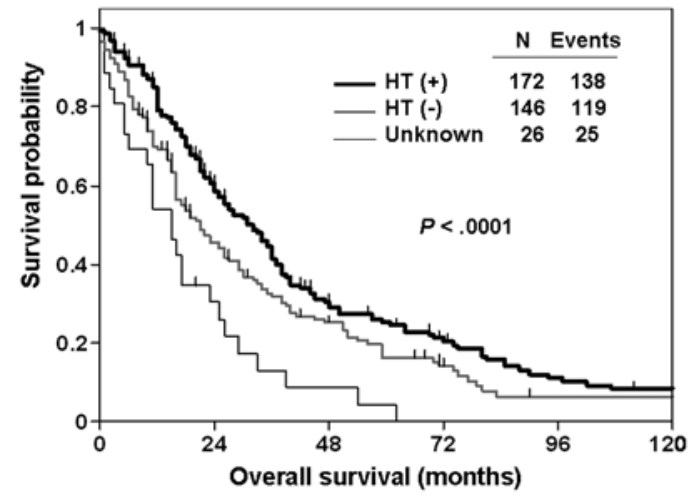

Figure 1. Kaplan-Meier curves of overall survival for MBC patients: (A) comparison of local surgery and no surgery; (B) comparison of younger $(\leq 50)$ and older patients $(\geq 51)$; (C) comparison of bone/soft tissue and visceral metastases; (D) comparison of hormone therapy and no hormone therapy. 


\section{A. Younger patients $(<50)$}

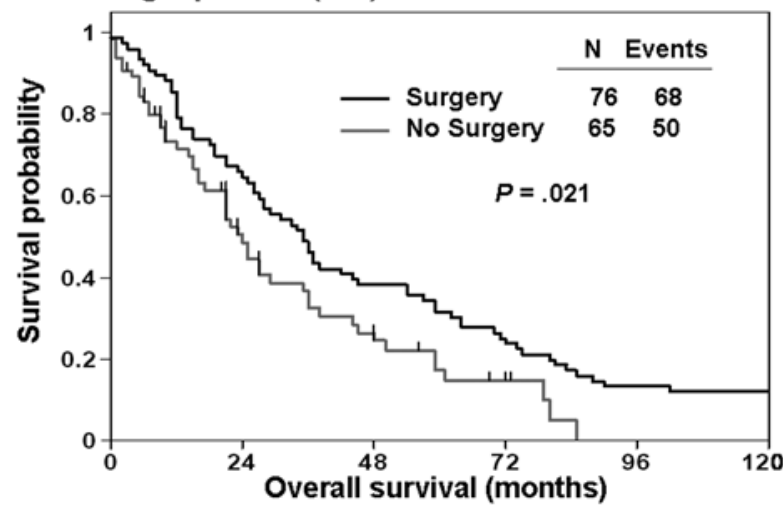

B. Older patients $(>51)$

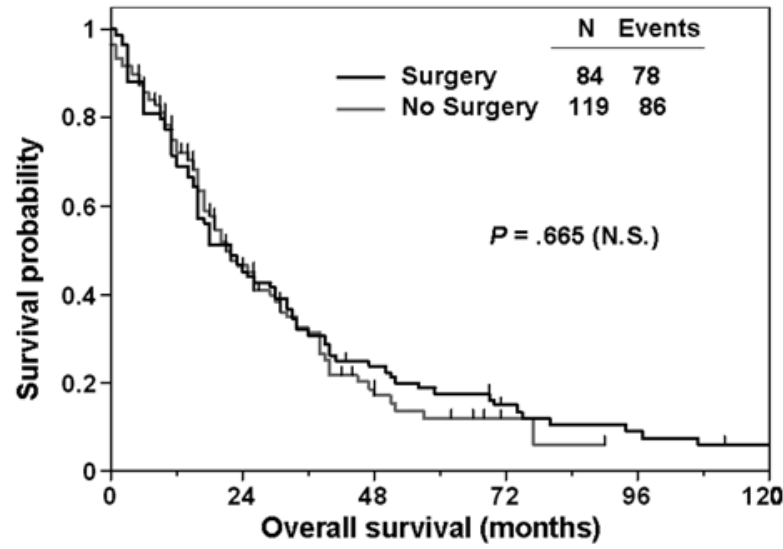

C. Bone/soft tissue metastases

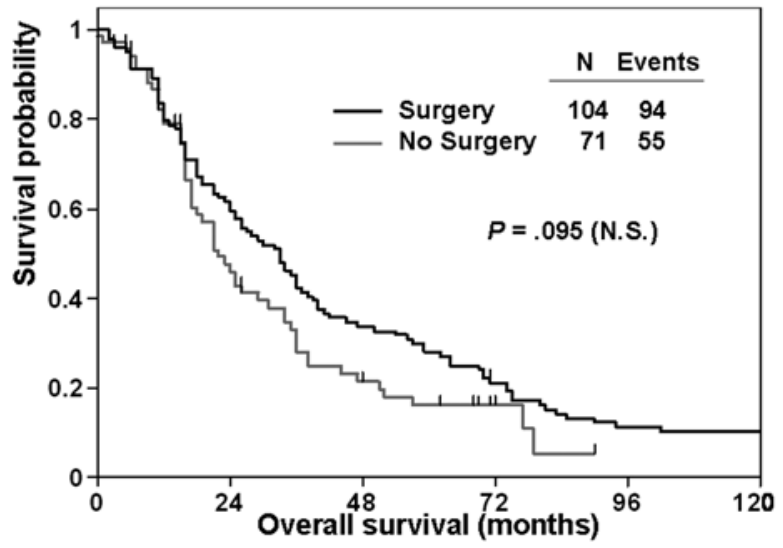

D. Visceral metastases

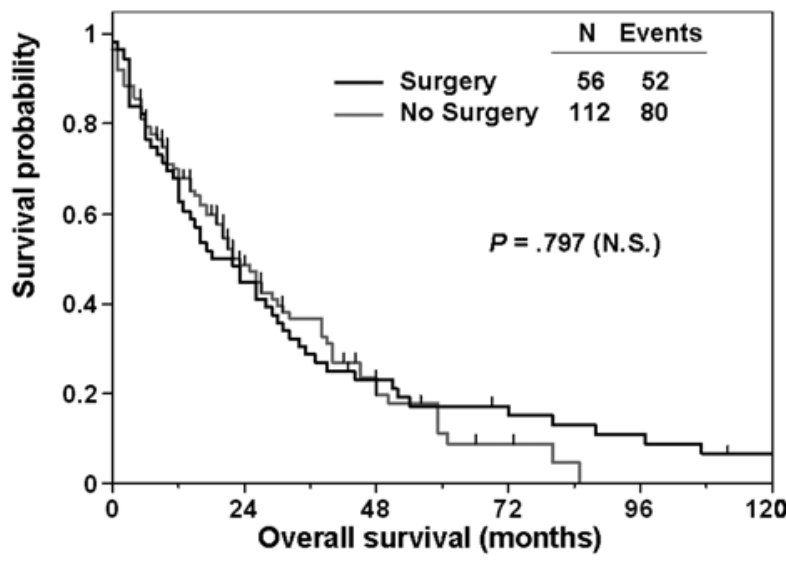

Figure 2. Kaplan-Meier curves of overall survival in the local surgery and no surgery groups: (A) younger patients ( $\leq 50)$; (B) older patients $(\geq 51)$; (C) bone/soft tissue metastases; (D) visceral metastases.
Table II. Patient characteristics by surgery group.

\begin{tabular}{lrrr}
\hline Parameters & $\begin{array}{c}\text { No. of pts }(\%) \\
\text { no surgery }\end{array}$ & Surgery & P-value \\
& & & \\
Age, median (range) & & & \\
$<50$ & $119(59)$ & $84(41)$ & \\
$>51$ & $65(46)$ & $76(54)$ & 0.02 \\
Period of diagnosis & & & \\
$1962-1980$ & $8(12)$ & $58(88)$ & \\
$1981-1990$ & $8(13)$ & $54(87)$ & \\
$1991-2000$ & $53(55)$ & $53(45)$ & \\
$2001-2007$ & $115(96)$ & $5(4)$ & $<0.0001$ \\
Clinical T stage & & & \\
T1 & $12(57)$ & $9(43)$ & \\
T2 & $29(48)$ & $31(52)$ & \\
T3 & $23(43)$ & $30(57)$ & \\
T4 & $119(57)$ & $89(93)$ & 0.39 \\
Site of metastases & & & \\
Bone/soft tissue & $67(40)$ & $102(60)$ & \\
Visceral & $116(67)$ & $58(33)$ & $<0.0001$ \\
Hormonal therapy & & & \\
Yes & $84(49)$ & $88(51)$ & \\
No & $89(61)$ & $57(39)$ & \\
Unknown & $11(42)$ & $15(58)$ & 0.05 \\
\hline
\end{tabular}

impacting quality of life. Current anti-tumor drugs, such as the anthracyclines and taxanes, are quite effective, as are molecularly targeted drugs such as trastuzumab. Using these drugs, the response rate of patients with locally advanced breast cancer was $80-90 \%$; many primary breast cancers were reduced and resected in breast-conserving surgery $(8,9)$. Other effective agent with fewer side effects, such as aromatase inhibitors and oral 5-fluorouracil, can prevent further disease progression, keeping patients stable and maintaining their quality of life for extended periods. Therefore, the control and/or reduction of both primary and metastatic lesions using systemic therapies has improved the living conditions of patients with MBC.

Surgery for breast cancer has also become safer and less invasive with the advent of improved surgical techniques and diagnosis, such as breast-conserving surgery and sentinel lymph node biopsy (10-12). These surgeries have few complications. However, several intensive chemotherapies have destructive high-grade and long-term side effects. Moreover, chemotherapy needs to be continued. According to the Hortobagyi algorithm, minimal surgery performed early in the treatment of MBC does not negatively impact quality of life. We need to evaluate prospectively the difference between local surgery and intensive chemotherapy. As studies have also demonstrated that local surgery for MBC avoids uncontrolled chest disease (13), local surgery for MBC should be discussed with patients as early as possible. 
We evaluated the efficacy of local surgery in MBC patients treated at $\mathrm{NCCH}$ through a comprehensive chart review. The medical oncologists currently follow the principles of MBC treatment outlined by the current National Cancer Institute (NCI) guidelines (1). Only rarely do MBC patients undergo local surgery; the aims of such surgeries were to avoid uncontrolled chest disease late in treatment. From 1960 to 1990, however, early primary tumor resection was significantly more common because there were far fewer effective drugs. In addition, there were patients who were discovered to have MBC immediately after surgery for the primary lesion, because in those days we could not examine and get the results of tests for metastases immediately. Therefore, it was more common for MBC patients diagnosed in previous decades to undergo local surgery. While this retrospective cohort study has several selection biases, the results demonstrate an efficacy of local surgery in MBC similar to previous studies.

Moreover, in our data many patients with local surgery treated in the early period of the study when we could not use effective chemotherapy (taxane and/or anthracycline), these active local surgery prolonged survival. However, in previous studies the time of surgeries were unclear. The time of local surgery is important to decide and consider the strategy of treatment for MBC patients. We think that the active local surgeries which prolong survival and prevent uncontrolled chest disease should be performed relatively early because treatment after a series of chemotherapy and radiation therapy, primary lesion becomes large and a more invasive surgical procedure is needed. The less invasive surgery can be performed in the time when the effective chemotherapy makes the primary lesion smaller. We also examined the efficacy of early local surgery, however, analysis of patients receiving early surgery did not reach statistically significant levels.

Of other clinicopathological features, age at diagnosis and site of metastasis were significantly predictive of improved OS for MBC patients. As expected the overall survival of young patients or patients with bone metastases was longer than old patients or with visceral metastases. In additional analysis, there was a clear benefit of local surgery especially for younger patients $<50$ years old. In older patients, there was no survival benefit of local surgery. These results demonstrate the possibility to change the strategy of treatment for stage IV breast cancer by age. The difference reported in previous studies (3-6) was not significant for patients with bone and soft tissue metastasis compared with those with visceral metastases. Almost all long-term survivors who underwent local surgery were younger patients with bone and/or soft tissue metastases who went into complete remission following systemic therapy.

We previously reported that MBC patients who had complete remissions at metastatic sites following systemic therapy had a better prognosis in comparison to other patients (14). The number of controllable patients with a good prognosis will hopefully increase with the effective new antitumor drugs such as trastuzumab (8). In this study, it was difficult to establish a relationship between overall survival and hormonal therapy or hormone receptor expression because the data from patients treated at the beginning of the study period lacked sufficient information.
Herein we report that local surgery improved overall survival in MBC patients. This effect was especially notable in patients $<50$ years. In addition, patients with bone and/or soft tissue metastases had a better prognosis. In other metastatic cancer types, several studies have reported the efficacy of primary tumor debulking surgery (15-18). Almost all of these reports were retrospective studies; only one prospective report indicated a benefit of surgery in renal cancer patients (17). In addition, there is a report that selfseeding from primary cancer decides the incidence and growth of metastatic disease (19). However, the biological mechanisms underlying such a response remain unclear.

The aim of local surgery was to avoid uncontrolled chest disease late in treatment. However, in late period of treatment local surgery becomes relatively invasive for complete resection because the primary tumor is regrowing. We think the primary tumor can be removed less invasively in early period of treatment when the primary tumor is reduced by effective systemic therapy. Additional cases and prospective studies are required to investigate the biological underpinnings of treatment to better understand the appropriate treatment for metastatic cancer.

\section{References}

1. National Cancer Institute: The treatment of primary breast cancer: Management of local disease. NIH Concensus Development Conference Summary 2: 1-3, 1994.

2. National Institutes of Health Consensus Development Panel: Consensus statement: treatment of early stage breast cancer. J Natl Cancer Inst Monogr 11: 1-5, 1992.

3. Rapiti E, Verkooijen HM, Vlastos G, et al: Complete excision of primary breast tumor improves survival of patients with metastatic breast cancer at diagnosis. J Clin Oncol 24: 2743-2749, 2006.

4. Gnerlich J, Jeffe DB, Deshpande AD, Beers C, Zander C and Margenthaler JA: Surgical removal of the primary tumor increases overall survival in patients with metastatic breast cancer: analysis of the 1988-2003 SEER Data. Ann Surg Oncol 14: 2187-2194, 2007

5. Babiera GV, Rao R, Feng L, et al: Effect of primary tumor extirpation in breast cancer patients who present with Stage IV disease and an intact primary tumor. Ann Surg Oncol 13: 776-782, 2005.

6. Khan SA, Stewart AK and Morrow M: Does aggressive local therapy improve survival in metastatic breast cancer? Surgery 132: 620-627, 2002.

7. Hortobagyi GN: Treatment of breast cancer. N Engl J Med 339: 974-984, 1998.

8. Buzdar AU, Ibrahim NK, Francis D, et al: Significantly higher pathologic complete remission rate after neoadjuvant therapy with trastuzumab, paclitaxel, and epirubicin chemotherapy: results of a randomized trial in human epidermal growth factor receptor 2-positive operable breast cancer. J Clin Oncol 23: 3676-3685, 2005.

9. Bear HD, Anderson S, Smith RE, et al: Sequential preoperatrive or postoperative docetaxel added to preoperative doxorubicin plus cyclophosphamide for operable breast cancer: National Surgical Adjuvant Breast and Bowel Project Protocol B-27. J Clin Oncol 24: 1-9, 2006.

10. Turner L, Swindell R, Bell WGT, Hartley RC, Tasker JH Wilson WW, et al: Radical versus modified radical mastectomy for breast cancer. Ann R Coll Surg Engl 63: 239-243, 1981.

11. Fisher B, Jeong J-H, Anderson S, Bryant J, Fisher ER and Wolmark N: Twenty-five-year follow up of a randomized trial comparing radical mastectomy, total mastectomy, and total mastectomy followed by irradiation. N Engl J Med 347: 567-575, 2002.

12. Veronesi U, Cascinelli N, Mariani L, Greco M, Saccozzi R, Luini A, et al: Twenty-year follow-up of a randomized study comparing breast-conserving surgery with radical mastectomy for early breast cancer. N Engl J Med 347: 1227-1232, 2002. 
13. Dalberg K, Liedberg A, Johansson U and Rutqvist LE: Uncontrolled local disease after salvage treatment for ipsilateral breast tumour recurrence. Eur J Surg Oncol 29: 143$154,2003$.

14. Yamamoto N, Katsumata N, Watanabe T, Omuro Y, Ando M, Narabayashi M and Adachi I: Clinical characteristics of patients with metastatic breast cancer with complete remission following systemic treatment. Jpn J Clin Oncol 28: 368-373, 1998.

15. Rosen SA, Buell JF, Yoshida A, et al: Initial presentation with stage IV colorectal cancer: how aggressive should we be? Arch Surg 135: 530-534, 2000.

16. Martin R, Paty P, Fong Y, et al: Simultaneous liver and colorectal resections are safe for synchronous colorectal liver metastasis. J Am Coll Surg 197: 233-241, 2003.
17. Flanigan RC, Salmon SE, Blumenstein BA, et al: Nephrectomy followed by interferon alfa- $2 b$ compared with interferon alfa- $2 b$ alone for metastatic renal cell cancer. N Engl J Med 345: 1655-1659, 2001.

18. Chi DS, Eisenhauer EL, Lang J, et al: What is the optimal goal of primary cytoreductive surgery for bulky stage IIIC epithelial ovarian carcinoma (EOC)? Gynecol Oncol 103: 559-564, 2006.

19. Norton L and Massague J: Is cancer a disease of self-seeding? Nat Med 12: 875-878, 2006. 